\title{
Aspectos biométricos e morfológicos de frutos e sementes de Schinopsis brasiliensis
}

\author{
Jania Claudia Camilo dos SANTOS ${ }^{1 *}$, Dayane Mércia Ribeiro SILVA ${ }^{1}$, Renato Nunes COSTA ${ }^{1}$, \\ Carlos Humberto da SILVA², Wilton da Silva SANTOS ${ }^{2}$, Flávia de Barros Prado MOURA ${ }^{1}$, José Vieira SILVA ${ }^{1}$ \\ ${ }^{1}$ Programa de Pós-Graduação em Agricultura e Ambiente, Universidade Federal de Alagoas, Arapiraca, AL, Brasil. \\ ${ }^{2}$ Departamento de Agronomia, Unidade de Ciências Agrárias, Universidade Federal de Alagoas, Arapiraca, AL, Brasil. \\ *E-mail: janya_claudia@yahoo.com
}

Recebido em março/2017; Aceito em dezembro/2017.

\begin{abstract}
RESUMO: O objetivo deste trabalho foi avaliar a biometria e morfologia de $S$. brasiliensis, visando identificar características que auxiliem no uso e propagação dessa espécie da Caatinga em programa de restauração ambiental. Os dados biométricos foram obtidos a partir de 1000 frutos e 1000 sementes. Foram medidos o comprimento, largura, espessura e massa de frutos e sementes. Os dados foram ajustados utilizando a estatística descritiva e teste de Shapiro-Wilk. Para a descrição morfológica dos frutos, foram relatadas observações sobre os aspectos externos e internos do pericarpo, consistência da casca, coloração, brilho, forma e deiscência. Já as descrições morfológicas externa das sementes foram feitas observações sobre consistência, coloração, textura, forma e hilo. A caracterização biométrica de frutos e sementes de S. brasiliensis, possibilitou verificar a variação existente entre as classes biométricas através de histograma de frequência. Os frutos apresentam morfologia completa, em forma de sâmara, indeiscente e monospérmico. As sementes foram caracterizadas em oblongas e reniformes, envolvidas pelo endocarpo, resultando no pirênio, sendo esta a camada que dificulta o processo de embebição de água, a qual antecede a germinação, no entanto, esta camada é capaz de promover maior longevidade as sementes.
\end{abstract}

Palavras-chave: análises biométricas, características morfológicas, sementes florestais, Caatinga.

\section{Biometric and morphological aspects of the fruits and seeds of Schinopsis brasiliensis}

\begin{abstract}
The objective of this work was to evaluate the biometry and morphology of S. brasiliensis, aiming to identify traits that help in the use and propagation of this Caatinga species in an environmental restoration program. Biometric data were obtained from 1000 fruits and 1000 seeds. The length, width, thickness and mass of fruits and seeds were measured. Data were adjusted using descriptive statistics and Shapiro-Wilk test. For the fruits morphological description, observations on the external and internal traits of the pericarp, peel consistency, coloration, brightness, shape and dehiscence were reported. Already the external morphological descriptions of the seeds were made on consistency, color, texture, shape and thread. The biometric characterization of fruits and seeds of $S$. brasiliensis made it possible to verify the variation between the biometric classes through frequency histogram. The fruits presented complete morphology, in the form of a samara, indiscriminate and monospermic. The seeds were characterized in oblong and reniform, surrounded by the endocarp, resulting in the pyrenium, which is the layer that hinders the process of water imbibition, which precedes the germination, however this layer is able to promote greater longevity of the seeds.
\end{abstract}

Keywords: biometric analyzes, morphological traits, forest seeds, Caatinga.

\section{INTRODUÇÃO}

O bioma Caatinga é um dos menos estudados e mais complexos biomas brasileiros, cujos fatores topográficos relacionados à vegetação típica da biodiversidade brasileira ainda é pouco conhecida, inclusive no campo da ciência, onde o interesse pelo bioma é recente, e o conhecimento produzido ainda é restrito (BISPO et al., 2010; SANTOS et al., 2010; AVANCINI; TEGA, 2013).

Diante da importância da preservação, a legislação Brasileira, através das Portarias do IBAMA no 83 (26/09/91) e $n^{\circ}$. 37-N (3/04/1992), lista várias espécies da flora e fauna da Caatinga, como ameaçadas de extinção, dentre as espécies vulneráveis, encontra-se a Schinopsis brasiliensis Engl., vulgarmente conhecida como baraúna ou braúna (DANTAS et al., 2008).

Informações voltadas a melhores condições de propagação são necessários para recomposição de áreas degradadas. Plantios conservacionistas de recuperação investem na produção de mudas florestais nativas, que apresentem boa qualidade fisiológica, selecionadas inclusive por tamanho e massa das sementes (VARELA et al., 2005; NÓBREGA et al., 2008; SANTOS et al., 2012, SILVA et al., 2014; MATOS et al., 2015; PIRES NETO et al., 2016).

Os aspectos biométricos e morfológicos de frutos e sementes são fundamentais para fornecer subsídios capazes de auxiliar nas informações de propagação da espécie (MATHEUS; LOPES, 2007; GONÇALVES et al., 2013; FERREIRA; BARRETO, 2015; GOMES et al., 2016). Na estratégia de maximizar e favorecer a uniformidade emergencial de plântulas, para obtenção de mudas superiores em qualidade e vigor (CARVALHO; NAKAGAWA, 2000).

Considerando que existem poucos estudos utilizando esta espécie, principalmente relacionados à caracterização biométrica, ainda não há uma classificação referente ao 
padrão em tamanho e massa de frutos e sementes desta espécie. Diante dos relatos supracitados, o objetivo deste trabalho foi descrever informações referentes aos aspectos biométricos e morfológicos de frutos e sementes de $S$. brasiliensis. Estas informações poderão auxiliar na indicação desta espécie em programas de reposição de áreas degradadas ocasionadas pela ação antrópica.

\section{MATERIAL E MÉTODOS}

Os frutos de Braúna (Schinopsis brasiliensis) foram coletados em aproximadamente 20 árvores matrizes distanciadas por no mínimo $50 \mathrm{~m}$, localizado no município de Olho D'água do Casado, alto Sertão de Alagoas ( $9^{\circ} 30^{\prime} 03^{\prime}$ ' S e 3749'56" W, $230 \mathrm{~m}$ ). A condução do trabalho foi realizada no Laboratório de Fisiologia Vegetal, da Universidade Federal de Alagoas, Campus Arapiraca, AL (9॰45'09'S; 36³9'40"W e $324 \mathrm{~m}$ ).

Os dados biométricos foram obtidos a partir de 1000 frutos e 1000 sementes, para obtenção do comprimento, largura, espessura e massa. Os frutos e sementes foram mensurados quanto ao comprimento, aferido da base de maior distância, em sementes e em frutos, até a inserção do pecíolo, largura e espessura medidos na linha mediana central, utilizando paquímetro digital e fita milimétrica, sendo posteriormente, pesados em balança analítica com precisão de $0,001 \mathrm{~g}$, para obtenção do peso médio.

Para análise dos dados foram realizados histogramas de distribuição de frequência. O ajuste de distribuição estatística e estatística descritiva compreenderam os valores mínimos, máximos, média amostral distribuídos em medidas de posição, assim como as medidas de dispersão que compreendem o desvio padrão, coeficiente de variação, de assimetria e curtose, além do teste de Shapiro-Wilk que relatam quanto à aderência dos dados as distribuições, as análises foram possíveis com o auxílio do programa estatístico SISVAR ${ }^{\circledR}$ (FERREIRA, 2014).

Para a descrição morfológica dos frutos, foram relatadas observações sobre os aspectos externos e internos do pericarpo, consistência da casca, coloração, brilho, forma e deiscência. Já a descrição morfológica externa das sementes foi relatada observações sobre consistência, coloração, textura, forma e hilo.

As observações para caracterização morfológica dos frutos e sementes foram realizadas com auxílio de lupa de mesa estereoscópica, com luz acoplada e a fotodocumentação realizada em máquina digital Cannon 1400, para posterior recalque das ilustrações e estruturas visualizadas.

\section{RESULTADOS}

A análise descritiva revelou aderência estatística entre as variáveis analisadas de maneira representativa. Os valores mínimos e máximos foram referenciados pela média aritmética como medida de tendência central de variabilidade entre os dados biométricos de frutos e sementes.

Os dados foram satisfatoriamente ajustados pelas medidas de dispersão (assimetria e curtose) e correlacionados para comprimento, largura, espessura e massa de frutos e sementes de $S$. brasiliensis, revelando através do teste Shapiro-Wilk que os dados não se ajustam a distribuição normal $(\mathrm{p}<0,05)$ (Tabela 1).

O coeficiente de variação biométrica de frutos de $S$. brasiliensis, baseados nos valores do desvio padrão e média, apresentou $8,18 \%$ para comprimento, com variação para largura, espessura e massa de 43,94; 37,74 e 15\%, respectivamente. Para a caracterização biométrica das sementes, verificou-se uma variação de $8,06 \%$ para comprimento, de $9,51 \%$ para largura, $13,24 \%$ para espessura e 23,08\% para massa das sementes (Tabela 1 ).

Tabela 1. Estatística descritiva dos dados biométricos de frutos e sementes, analisados para comprimento (C), largura (L), espessura (E) e massa (M) de Schinopsis brasiliensis.

Table 1. Descriptive statistics of the fruits and seeds biometric data, analyzed for length (L), width (W), thickness (T) and mass (M) of Schinopsis brasiliensis.

\begin{tabular}{|c|c|c|c|c|}
\hline \multirow{2}{*}{ Estatística } & \multicolumn{4}{|c|}{ FRUTOS } \\
\hline & $\mathrm{C}$ & $\mathrm{L}$ & $\mathrm{E}$ & $\mathrm{M}$ \\
\hline & \multicolumn{3}{|c|}{$(\mathrm{mm})-$} & $--(\mathrm{g})--$ \\
\hline Mínimo & 12,10 & 6,70 & 4,50 & 0,02 \\
\hline Máximo & 56,30 & 125,00 & 61,00 & 0,29 \\
\hline Média & 32,52 & 12,22 & 6,65 & 0,20 \\
\hline Desvio-Padrão & 2,66 & 5,37 & 2,51 & 0,03 \\
\hline Assimetria ${ }^{(1)}$ & 1,02 & 18,31 & 14,13 & $-0,40$ \\
\hline Curtose $+3^{(2)}$ & 2,44 & 3,77 & 2,59 & 4,67 \\
\hline \multirow[t]{2}{*}{ Shapiro-Wilk ${ }^{(3)}$} & $0,00^{*}$ & $0,00^{*}$ & $0,00^{*}$ & $0,00 *$ \\
\hline & \multicolumn{4}{|c|}{ SEMENTES } \\
\hline Mínimo & 7,60 & 5,30 & 2,30 & 0,02 \\
\hline Máximo & 14,60 & 10,00 & 7,70 & 0,23 \\
\hline Média & 11,42 & 7,57 & 3,55 & 0,13 \\
\hline Desvio-Padrão & 0,92 & 0,72 & 0,47 & 0,03 \\
\hline Assimetria $^{(1)}$ & $-0,05$ & $-0,08$ & 1,97 & $-0,21$ \\
\hline Curtose $+3^{(2)}$ & 3,57 & 2,72 & 5,17 & 3,45 \\
\hline Shapiro-Wilk ${ }^{(3)}$ & $0,00^{*}$ & $0,00^{*}$ & $0,00^{*}$ & $0,00^{*}$ \\
\hline
\end{tabular}

${ }^{(1)}$ Assimetria difere de zero, pelo teste $t$, ao nível de $5 \%$ de probabilidade. ${ }^{(2)}$ Curtose difere de três, pelo teste t, ao nível de $5 \%$ de probabilidade. (3) $*$ Significativo a $5 \%$ de probabilidade.

Os resultados da estatística descritiva podem ser ainda mais refinados e precisos quando se observa os valores do desvio padrão amostral, os quais apresentaram variação entre os valores desde 0,03 a 5,37, indicando que os dados biométricos de frutos e sementes de $S$. brasiliensis foram apresentados com precisão dentro de uma ampla população amostral (Tabela 1).

Em posse da variação existente entre a massa dos frutos e sementes de $S$. brasiliensis, é possível tornar esta informação ainda mais precisa, ao dividir o peso médio das sementes pelo peso médio dos frutos, constatando que as sementes representam $65 \%$ da alocação de biomassa total, restando apenas 35\% para a casca dos frutos (Tabela 1).

Pode-se considerar que a maior quantidade de fotoassimilados foi translocado para as sementes, proporcionando uma maior quantidade de reserva representada pela massa.

Ao analisar os dados de simetria da curva, pode-se observar distribuição assimétrica negativa para massa dos frutos, assim como para comprimento, largura e massa das sementes, apresentando as demais variáveis com assimetria positiva.

Quanto à curtose, os dados são próximos ou superiores a três, indicando distribuição mesocúrtica e platicúrtica em frutos e sementes. Com a obtenção do teste de Shapiro-Wilk comprova-se a significância entre os dados biométricos amostrais, os quais confirmam a não normalidade dos dados (Tabela 1; Figura 1).

Para comprimento dos frutos observou-se curva simétrica, enquanto que a largura e espessura apresentam 
assimetria à direita, com distribuição assimétrica à esquerda para massa (Figura 1). Nas sementes o comprimento, largura e massa, apresentam polígono de frequência simétrico, com curva assimétrica à direita na espessura (Figura 2).

A espessura das sementes de S. brasiliensis apresentou distribuição platicúrtica (Tabela 1, Figura 2C), enquanto que as demais características apresentaram distribuição leptocúrtica (Tabela 1, Figura 2) conforme o coeficiente de curtose (Curtose $>3$ ).

Ampla distribuição foi observada entre as classes de frequência dos frutos, em que $96 \%$ apresentam comprimento médio em torno de 27,89 a $37,36 \mathrm{~mm}$, cerca de $99 \%$ apresentam largura de 6,70 a $15,15 \mathrm{~mm}$, e $97 \%$ com espessura de 4,50 a $8,54 \mathrm{~mm}$, e massa de $93 \%$ entre 0,14 a $0,27 \mathrm{~g}$ (Figura 1).
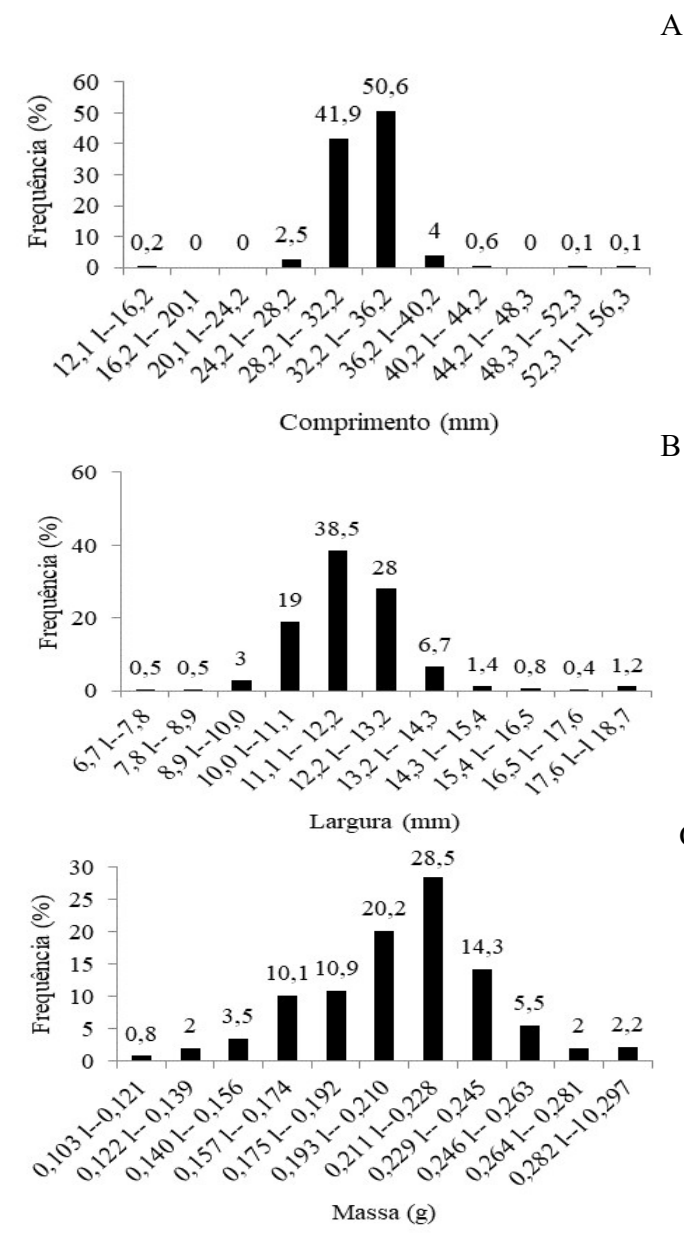

C

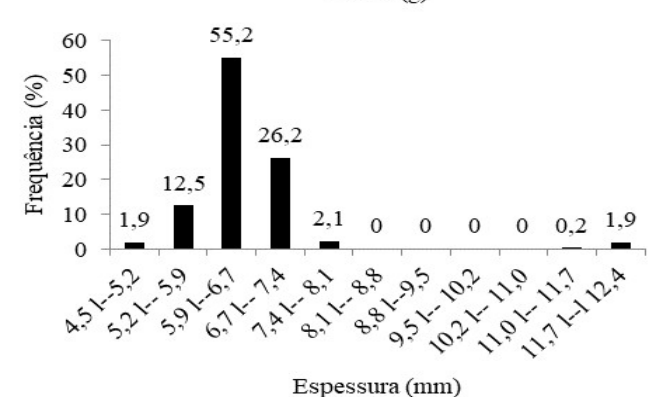

Figura 1. Classes de distribuição de frequência para comprimento (A), largura (B), espessura (C) e massa (D) de frutos de Schinopsis brasiliensis.

Figure 1. Frequency distribution classes for length (A), width (B), thickness (C) and mass (D) of Schinopsis brasiliensis fruits.
A variação existente entre as classes de distribuição das sementes, correspondem cerca de $97 \%$ para comprimento, entre 9,10 a 13,60 mm, 98\% com largura nas classes de 5,97 a $8,99 \mathrm{~mm}, 99 \%$ com espessura em torno de 2,69 a 4,61 mm, e ainda $99 \%$ com peso distribuídos entre as classes de 0,07 a 0,19 (Figura 2).

Entre as diferentes partes que compõem os frutos de $S$. brasiliensis, podemos diferenciá-las de acordo com a alocação de biomassa de cada estrutura, de forma a obter valores com maior precisão entre os dados amostrados.

Como a casca correspondente a $32 \%$ da alocação de biomassa total, e as sementes são responsáveis por $68 \%$ dos valores amostrais, pode-se constar, que do total de sementes obtidas dos frutos, cerca de $93 \%$ apresentam peso médio considerado satisfatório para a espécie (Tabela 1).

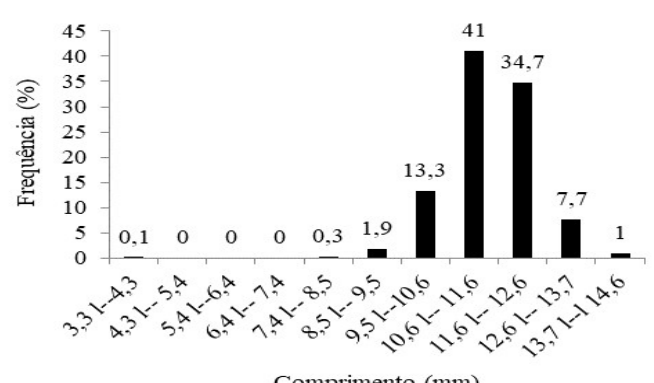

A

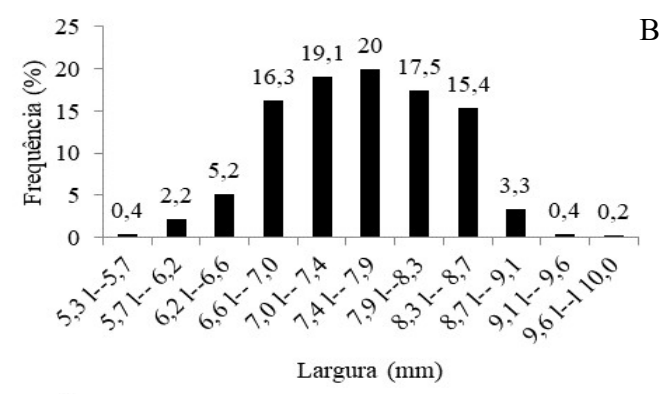

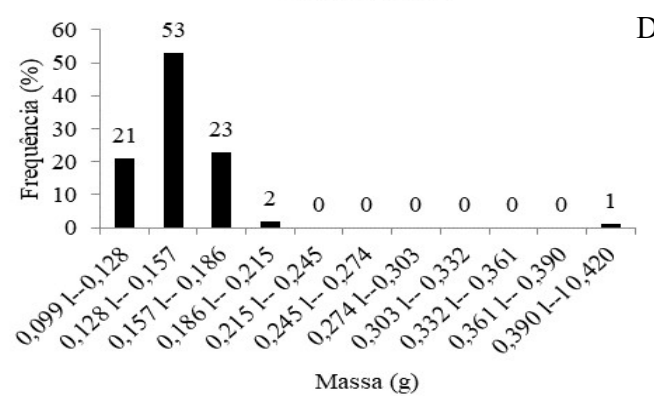

Figura 2. Classes de distribuição de frequência para comprimento (A), largura (B), espessura (C) e massa (D) de sementes de Schinopsis brasiliensis.

Figure 2. Frequency distribution classes for length (A), width (B), thickness (C) and mass (D) of Schinopsis brasiliensis seeds. 
Estas são informações precisas sobre as características dessa espécie provenientes do Sertão Nordestino, em que mesmo sobre condições ambientais pouco favoráveis, foram capazes de utilizar suas reservas para formação de frutos, com a finalidade de propagação.

Quanto aos aspectos morfológicos de S. brasiliensis, estes são apresentados em estruturas descritivas com vista lateral, dorsal e frontal de frutos e estrutura lateral da semente desidratada (Figura 3).

Quanto aos aspectos morfológicos de S.brasiliensis, os frutos são tipo sâmara por serem secos, indeiscentes, monospérmicos com pericarpo membranoso, mas sem aderência à semente, com mesocarpo esponjoso e endocarpo lenhoso do tipo ósseo sendo impermeável à água contém apenas uma única semente por fruto, com brilho opaco e superfície lisa, oblongo, levemente reticulado, com base aguda, possui estrutura falciforme, leves e alados. A coloração varia de acordo com o grau de maturação dos frutos que modifica de verde à coloração amarronzada quando os frutos estão maduros (Figura 3).

\section{DISCUSSÃO}

Os frutos e sementes apresentaram uma ampla variação nos aspectos biométricos. Isto já era esperado considerando que estas espécies estão em ambiente natural na Caatinga nordestina. No entanto, quando esta variação é muito baixa pressupõe-se que as matrizes estão presentes em ambiente com vegetação primária (RODRIGUES et al., 2015).

A variação existente entre os frutos está relacionada às condições em que as árvores matrizes estão submetidas, principalmente no período de floração e frutificação, as quais podem promover mudanças que decorrem da variabilidade genética ou plasticidade fenotípica (VIEIRA; GUSMÃO, 2008).

Esta variabilidade em classes biométricas diferentes de frutos e sementes está relacionada à partição de biomassa e/ou condições nutricionais da planta mãe durante o enchimento dos frutos. Tal capacidade em fornecer nutrientes para o enchimento das sementes está relacionado à maior quantidade de reserva da planta mãe, captados na translocação de fotoassimilados, e fornecidos as sementes durante a partição de biomassa (formação de frutos e sementes), recebendo assim, as melhores condições de viabilidade.

Estudos relacionados à caracterização biométrica desta espécie, ainda são escassos. No entanto, estudos evidenciam que diásporos de maior tamanho apresentam maior sucesso germinativo, crescimento e sobrevivência da plântula, por se originarem de plântulas competitivamente superiores e consequentemente mais vigorosas (GONÇALVES et al. 2013).

Os dados assimétricos quando apresentam maiores valores próximo ao mínimo, esta é considerada positiva, mas quando estes valores estão próximos ao máximo, esta é considerada assimétrica negativa. A distribuição assimétrica entre frutos e sementes de Melanoxylon brauna foram estudados por Silva et al. (2013), os quais constataram a existência de distribuição assimétrica e platicúrtica para os frutos, e as sementes com largura em assimetria negativa, com comprimento e espessura com assimetria positiva.

Os frutos apresentam assimetria positiva e curtose elevada, sendo considerada como leptocúrtica, nos parâmetros avaliados como comprimento, largura e espessura. Em estudos realizados por Silva et al. (2013) em Melanoxylon brauna, ao avaliarem as características biométricas de sementes, verificaram que o comprimento e espessura possuíam distribuição assimétrica à direita, enquanto que, para a largura, a distribuição assimétrica foi à esquerda, e os frutos com distribuição assimétrica à direita.

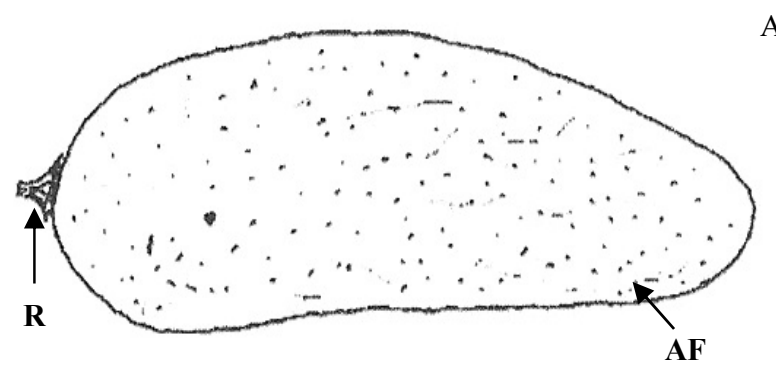

B
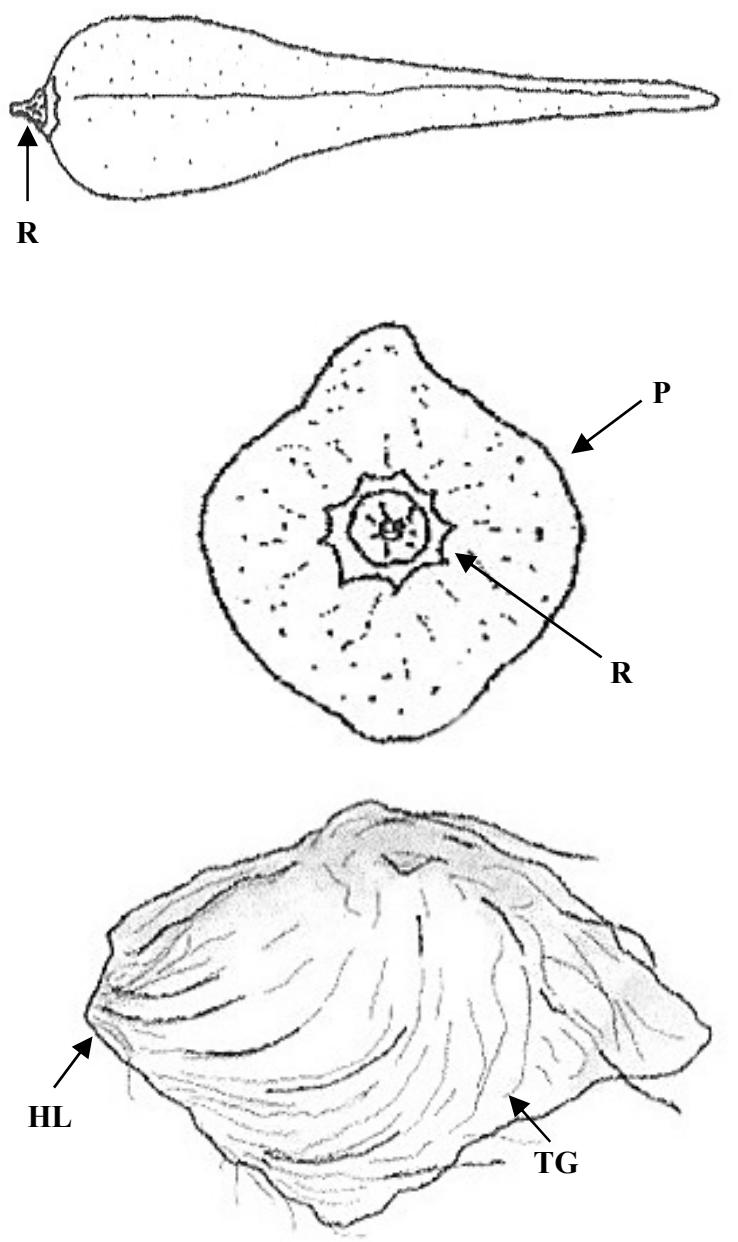

C

$\mathrm{D}$

Figura 3. Caracterização morfológica com vista lateral (A), vista dorsal (B), e vista frontal (C) de fruto, e semente com vista lateral desidratada (D) de Schinopsis brasiliensis. Legenda: R: receptáculo; AF: ala do fruto; P: pericarpo; HL: hilo; TG: tegumento.

Figure 3. Morphological characterization with lateral view (A), dorsal view (B), and frontal view (C) of fruit, and seed with lateral view dehydrated (D) of Schinopsis brasiliensis. Legend: R: receptacle; AF: fruit wing; P: pericarp; HL: hilum; TG: tegument.

É importante salientar que na distribuição leptocúrtica os dados estão aglomerados no centro da distribuição, apresentando assim, menor amplitude em relação à espessura das sementes. Enquanto que, na distribuição platicúrtica, a 
frequência das variáveis são mais achatadas do que a curva normal, ou seja, há maior amplitude de distribuição dos dados (VIEIRA; CARVALHO, 2009).

Os resultados encontrados neste trabalho corroboram com os encontrados por Dias et al. (2007) os quais constataram que os frutos de $S$. brasiliensis, avaliados de uma amostragem de 200 frutos coletados em 10 árvores matrizes, apresentam em média $30,9 \mathrm{~mm}$ de comprimento e $11,76 \mathrm{~mm}$ de largura, com sementes de $12,38 \mathrm{~mm}$ de comprimento e $8,73 \mathrm{~mm}$ de largura, apresentando uma média amostral entre as árvores da região.

Estudos que consideram a biometria e morfologia de espécies por região, são de fundamental importância, para diferenciação entre espécies de um mesmo gênero. A obtenção de lotes homogêneos é permitido pela classificação das sementes quanto ao peso, sendo esta uma característica fundamental para estudiosos em tecnologia de sementes.

Os aspectos morfológicos de $S$. brasiliensis estão de acordo com o trabalho realizado por Prado et al. (1996), para a descrição desta espécie. Segundo Nogueira et al. (2010), os frutos de Dalbergia cearensis por serem sâmaras, assim como relatado neste trabalho, se originam de ovário monocarpelar com expansão em forma de asa na semente, como uma maneira planar no mínimo uma curta distância. Dessa forma, segundo Barroso et al. (1999) a morfologia alada do fruto tipo sâmara está relacionado como forma de dispersão anemocórica, em que as sementes conquistam novos ambientes por fatores naturais.

De certa forma, a dispersão anemocórica é uma característica de muitas espécies da caatinga. Isto porque, sementes que apresentam leve estrutura de dispersão são capazes de atingirem longas distâncias, aumentando assim, as chances de sobrevivência e perpetuação durante as estações secas ou chuvosas (GRIZ; MACHADO, 2001). Esta característica é vantajosa para as espécies da Caatinga, pois permite que as unidades dispersas a longas distâncias possuam maiores chances de sobrevivência, não sendo alvos de patógenos e predadores (NOGUEIRA et al., 2010).

Quanto às características morfológicas, as sementes são oblongas e reniformes, ou seja, com formato de rim, apresentando funículo alongado, consistência rígida, textura lisa com pequenas reentrâncias e saliências, sem brilho, com tonalidade amarela clara, com hilo visível a olho nu, localizado na região basal. Envolvida pelo endocarpo, formando o pirênio (BARROSO et al., 1999), sendo a principal camada que resulta na impermeabilidade do tegumento das sementes (Figura 2D). De certa forma, essa camada proporciona maior longevidade às sementes, por dificultar a germinação mesmo em condições favoráveis, como uma estratégia de sobrevivência.

As sementes de $S$. brasiliensis apresentam tegumento duro (impermeável à água). Estudos referentes ao percentual médio de germinação realizado por Oliveira; Oliveira (2012), a fim de avaliar o melhor método na superação de dormência desta espécie, a qual é causada pela rigidez do endocarpo, foi constatado que o tratamento com água em temperatura elevada sobre as sementes, foi capaz de promover a germinação das sementes.

A intolerância do pericarpo à água quente sugere que este apresenta um papel fundamental na sobrevivência das sementes durante a estiagem na Caatinga (OLIVEIRA; OLIVEIRA, 2012). Sob condições de altas temperaturas na
Caatinga, onde o solo pode atingir $60^{\circ} \mathrm{C}$, as sementes podem ficar expostas por até 11 meses sem umidade e sobrevivem graças ao envoltório e às estruturas anexas, que exercem papel crucial na proteção, em face ao aquecimento e à perda de água (GUIMARÃES DUQUE, 1973; LABORIAU, 1983).

\section{CONCLUSÕES}

Os frutos e sementes $S$. brasiliensis apresentam grande variação nos aspectos biométricos. Com assimetria positiva em todos os parâmetros avaliados dos frutos.

As sementes apresentaram distribuição leptocúrtica para comprimento, largura e massa, com espessura platicúrtica.

Os frutos de $S$. brasiliensis apresentam morfologia completa com frutos em forma de sâmara, indeiscente e monospérmico, com apenas uma semente por fruto.

As sementes são oblongas e reniformes, envolvidas pelo endocarpo que resulta no pirênio, sendo esta a camada que dificulta a germinação e permite a longevidade desta espécie.

A classificação dos frutos e sementes por tamanho e massa irão auxiliar na pré-seleção de sementes desta espécie em programas de reposição de áreas degradadas ocasionadas pela ação antrópica.

\section{AGRADECIMENTOS}

À Fundação de Amparo à Pesquisa do Estado de Alagoas (FAPEAL). Ao Centro de Referência em Recuperação de Áreas Degradadas do Baixo São Francisco (CRAD), e UFAL - Arapiraca, pelo apoio logístico, técnico e financeiro.

\section{REFERÊNCIAS}

AVANCINI, M. M.; TEGA, G. Caatinga: um bioma entre a devastação e a conservação. ComCiência, n. 149, 2013.

BARROSO, G. M. MORIM, M. P.; PEIXOTO, A. L.; ICHASO, C. L. F. Frutos e sementes: morfologia aplicada à sistemática de dicotiledôneas. Viçosa, MG: Universidade Federal de Viçosa, 1999. 443 p.

BISPO, P. C.; VALERIANO, M. M.; KUPLICH, T. M. Relação da vegetação de caatinga com a condição geomorfométrica local. Revista brasileira de engenharia agrícola e ambiental, Campina Grande, v. 14, n. 5, p. 523-530, 2010. DOI: http://dx.doi.org/10.1590/S1415-43662010000500010

CARVALHO, N. M.; NAKAGAWA, J. Sementes: ciência, tecnologia e produção. 4. ed. Jaboticabal-SP: FUNEP, 2000. 588 p.

DANTAS, B. F.; SOARES, F. S. J.; LÚCIO, A. A.; ARAGÃO, C. A. Alterações bioquímicas durante a embebição de sementes de baraúna (Schinopsis brasiliensis Engl.). Revista Brasileira de Sementes, Londrina, v. 30, n. 2, 2008. DOI: http://dx.doi.org/10.1590/S0101-31222008000200027

DIAS, C. T. V.; SILVA, P. P.; KIILL, L. H. P. Morfologia e dispersão de frutos de Schinopsis brasiliensis (Anacardiaceae) na Reserva Legal do Projeto Salitre, Juazeiro-BA. In: JORNADA DE INICIAÇÃO CIENTÍFICA DA EMBRAPA SEMI-ÁRIDO, 2., 2007, Petrolina. Petrolina: Embrapa Semi-Árido, 2007. (Embrapa Semi-Árido. Documentos, 205). http: http://www.alice.cnptia.embrapa.br/handle/doc/160381

FERREIRA, D. F. Sisvar: a Guide for its Bootstrap procedures in multiple comparisons. Ciência $\mathbf{e}$ Agrotecnologia, Lavras, v. 38, n. 1, p. 109-112, 2014. 
DOI: 70542014000200001

FERREIRA, R. A.; BARRETTO, S. S. B. Morphological characterization of fruits, seeds, plantules and seedlings of brazil wood (Caesalpinia echinata LAMARCK). Revista Árvore, Viçosa, v. 39, p. 505-512, 2015. DOI: http://dx.doi.org/10.1590/0100-67622015000300011

GOMES, D. R.; ARAUJO, M. M.; NUNES, U. R.; AIMI, S. C. Biometry and germination of Balfourodendron riedelianum Eng. Eng.. Journal of Seed Science, Londrina, v. 38 , p. 187-194, 2016. DOI: http://dx.doi.org/10.1590/2317-1545v38n3159311

GONCALVES, L. G. V.; ANDRADE, F. R.; MARIMON JUNIOR, E. H.; SCHOSSLER, T. R.; MARIMON, B. S. Biometria de frutos e sementes de mangaba (Hancornia speciosa Gomes) em vegetação natural na região leste de Mato Grosso, Brasil. Revista de Ciências Agrárias, Lisboa, v. 36, p. 31-40, 2013.

GRIZ, L. M. S.; MACHADO, I. C. S. Fruiting phenology and seed dispersal syndromes in caatinga, a tropical dry forest in the northeast of Brazil. Journal of Tropical Ecology, v. 17, p. 303-321, 2001.

GUIMARÃES DUQUE, J. Solo e água no polígono das secas. 4.ed. Fortaleza: Ministério da Viação e Obras Contra as secas, 1973. 310p. (Publicação 154, Série I-A).

LABORIAU, L. G. A germinação das sementes. Washington: OEA, 1983. 174p. (OEA, Série de Biologia: Monografia, 24).

MATHEUS, M. T.; LOPES, J. C. Morfologia de frutos, sementes e plântulas e germinação de sementes de Erythrina variegata L. Revista Brasileira de Sementes, v.29, n.3, p.08-17, 2007.

MATOS, A. C. B.; ATAIDE, G. M.; BORGES, E. E. L. Physiological, physical, and morpho-anatomical changes in Libidibia ferrea ((Mart. ex Tul.) L.P. Queiroz) seeds after overcoming dormancy. Journal of Seed Science, v. 37, p. 26-32, 2015. DOI: http://dx.doi.org/10.1590/2317$1545 \mathrm{v} 37 \mathrm{n} 1140433$

NOBREGA, R. S. A.; PAULA, A. M.; BOAS, R. C. V.; NOBREGA, J. C. A.; MOREIRA, F. M. S. Parâmetros morfológicos de mudas de Sesbania virgata (Caz.) Pers e de Anadenanthera peregrina (L.) cultivadas em substrato fertilizado com composto de lixo urbano. Revista Árvore, Viçosa, v. 32, p. 597-607, 2008. DOI: http://dx.doi.org/10.1590/S0100-67622008000300020

OLIVEIRA, M. C. P.; OLIVEIRA, G. J. Superação da dormência de sementes de Schinopsis brasiliensis. Ciência Rural, Santa Maria, v. 38, n. 1, p. 251-254, 2008. DOI: https://dx.doi.org/10.1590/S010384782008000100042
PIRES NETO, P. A. F.; PIRES, V. C. M.; MORAES, C. B.; OLIVEIRA, L. M.; PORTELLA, A. C. F.; NAKAGAWA, J. Physiological ripening of Anadenanthera colubrina (Vellozo) Brenan seeds. Journal of Seed Science, Londrina, v. 38, p. 155-161, 2016. DOI: http://dx.doi.org/10.1590/2317$1545 \mathrm{v} 38 \mathrm{n} 2153112$

RODRIGUES, J. K.; MENDONÇA, M. S; GENTIL, D. F. O. Aspectos biométricos, morfoanatômicos e histoquímicos do pirênio de Bactris maraja (Arecaceae). Rodriguésia, Rio de Janeiro, v. 66, p. 75-85, 2015. DOI: http://dx.doi.org/10.1590/2175-7860201566105

SANTOS, M. V. F.; LIRA, M. A.; DUBEUX JUNIOR, J. C. B.; GUIM, A.; MELLO, A. C. L.; CUNHA, M. V. Potential of Caatinga forage plants in ruminant feeding. Revista Brasileira de Zootecnia, Viçosa, v. 39, p. 204215, 2010. DOI: http://dx.doi.org/10.1590/S151635982010001300023

SANTOS, P. L.; FERREIRA, R. A.; ARAGÃO, A. G.; AMARAL, L. A.; OLIVEIRA, A. S. Estabelecimento de espécies florestais nativas por meio de semeadura direta para recuperação de áreas degradadas. Revista Árvore, Viçosa, v. 36, p. 237-245, 2012. DOI: http://dx.doi.org/10.1590/S0100-67622012000200005

SILVA, A. L.; DIAS, D. C. F. S.; LIMA, L. B.; MORAIS, G. A. Methods for overcoming seed dormancy in Ormosia arborea seeds, characterization and harvest time. Journal of seed Science, Londrina, v. 36, n. 318-325, 2014. DOI: http://dx.doi.org/10.1590/2317-1545v36n31012

SILVA, M. S.; BORGES, E. E. L.; LEITE, H. G.; CORTE, V. B. Biometria de frutos e sementes de Melanoxylon brauna Schott. (Fabaceae-Caesalpinioideae). Cerne, Lavras, v. 19, n. 3, p. 517-524, 2013. DOI: http://dx.doi.org/10.1590/S0104-77602013000300020

VARELA, V. P.; COSTA, S. S.; RAMOS, M. B. P. Influência da temperatura e do substrato na germinação de sementes de itaubarana (Acosmium nitens (Vog.) Yakovlev) - Leguminosae, Caesalpinoideae. Acta Amazônica, Manaus, v. 35, p. 35-39, 2005. DOI: http://dx.doi.org/10.1590/S0044-59672005000100006

VIEIRA, F. A.; CARVALHO, D. Maturação e morfometria dos frutos de Miconia albicans (Swartz) Triana (Melastomataceae) em um remanescente de floresta estacional semidecídua montana em Lavras, MG. Revista Árvore, Viçosa, v. 33, n. 6, p. 1015-1023, 2009. DOI: http://dx.doi.org/10.1590/S0100-67622009000600004

VIEIRA, F. A.; GUSMÃO, E. Biometria, armazenamento de sementes e emergência de plântulas de Talisia esculenta Radlk. (Sapindaceae). Ciência e Agrotecnologia, Lavras, v. 32 , n. 4, p. 1073-1079, 2008. DOI: http://dx.doi.org/10.1590/S1413-70542008000400006 